\title{
Spirale nach unten oder produktive Krisen? Zur Geschichte politischer Entscheidungskrisen der europäischen Integration
}

\author{
Hartmut Kaelble*
}

Die Europäische Union steckt seit 2007 in einer schweren Krise. Die akute Phase dieser Krise scheint überwunden zu sein, aber niemand weiß, ob nicht eine falsche Entscheidung des Europäischen Rates oder einer nationalen europäischen Regierung, der Zusammenbruch einer großen Bank oder gewalttätige Konflikte in einem Mitgliedstaat eine Rückkehr der Krise auslösen. Diese Krise war nicht nur eine unglückselige Verbindung von Finanzkrise, wirtschaftlichem Wachstumseinbruch und staatlicher Schuldenkrise. Sie war auch eine schwere Krise der europäischen Integration, eine dramatische Herausforderung für die Entscheider im Europäischen Rat und in der Europäischen Kommission, eine Zeit ungewöhnlicher Spannungen zwischen den nördlichen und südlichen Mitgliedstaaten der Europäischen Union, ein globaler Vertrauensverlust der Europäischen Union und eine tiefe Vertrauenskrise der Bürger in die Europäische Union. In der gegenwärtigen Grauzone zwischen latenter Krisenanfälligkeit und abgeflauter, aber allen noch präsenter Krise erscheint es sinnvoll, diese Krise in die lange Geschichte der Krisen der europäischen Integration einzuordnen und zu fragen, was sich in der jüngsten wiederholt hat und was an ihr neu war.

Drei ganz unterschiedliche Interpretationen der Geschichte von Krisen der europäischen Integration wurden angesichts dieser Krise vorgetragen und auch in den politischen Auseinandersetzungen eingesetzt. In aller Regel wurden diese Interpretationen auch schon in früheren Krisen der europäischen Integration debattiert, erhielten aber in der akuten Phase der Krise eine besondere Aufmerksamkeit. Diese drei Narrative sollen nur als Einstieg benutzt werden, ohne auf die Geschichte dieser Debatte in Politik und Wissenschaft eingehen zu können.

Eine erste Gruppe von Beobachtern und Entscheidern hatte die Befürchtung, manchmal auch die Hoffnung, dass sich die Krisen der europäischen Integration in einer Spirale nach unten immer mehr verstärkten und Europa vor einer finalen Krise, vor einem Zusammenbruch der Währung oder gar der Europäischen Union stehen könnte. In manchen Beiträgen wurden verschiedene Szenarien des Zusammenbruchs und der europäischen Politik nach der Europäischen Union durchgespielt, meist als abschreckende Warnung vor einem Zusammenbruch, manchmal aber auch in der Hoffnung auf ein Ende der jetzigen Europäischen Union und eine Rückkehr zu souveräner nationaler Politik.

Eine zweite Gruppe von Beobachtern glaubte auch in dieser schweren Krise weiterhin an produktive Krisen der europäischen Integration. Nach dieser schon klassischen Vorstellung entstanden aus den bisherigen Krisen der europäischen Integration regelmäßig Integrationsfortschritte. Die europäische Integration benötigte nach aller historischen Erfahrung sogar Krisen, um weiter voranzukommen. Krisen der europäischen Integration waren in dieser Sicht produktiv. Auch von der aktuellen Krise werden weitere Integrationsschritte zu einer stärkeren europäischen Kontrolle der nationalen Haushalte und des europäischen Bankensystems erwartet.

\footnotetext{
* Prof. Dr. em. Hartmut Kaelble, Institut für Geschichtswissenschaften, Humboldt Universität zu Berlin.
} 
Manchmal wird noch eine dritte, eher zyklische Vorstellung vorgetragen. In dieser Vorstellung treten schwere Krisen der europäischen Integration alle 30 bis 40 Jahre auf. Jede Epoche, auch jede Generation von Europapolitikern erlebt ihre eigene schwere europäische Krise und versucht ihre eigenen Lösungen zu finden. Schwere Krisen werden manchmal als Scheitern der Europapolitik der vorhergehenden Generation gesehen und gleichzeitig als Anfang der Europapolitik einer neuen Generation. ${ }^{1}$

Erstaunlicherweise sind die bisherigen Krisen der europäischen Integration in ihrer Gesamtheit nie daraufhin untersucht worden, ob sie eher eine Spirale nach unten waren, ob sie die europäische Integration immer weiter voranbrachten oder ob sie sich zyklisch entwickeln. Einzelne europäische Krisen sind allerdings von Historikern und Politikwissenschaftlern gründlich erforscht worden. Das gilt vor allem für die Krisen in den 1950er und 1960er Jahren. ${ }^{2}$ Zum Rückgang der Unterstützung der Europäischen Union in der öffentlichen Meinung seit 1990 gibt es zudem eine interessante, überwiegend politikwissenschaftliche Debatte. $^{3}$

1 Vgl. als jüngere Beispiele aus der Wissenschaft: Cyril Gläser/Wolfgang Wessels: Die Europapolitik in der wissenschaftlichen Debatte, in: Jahrbuch der Europäischen Integration 2012, Baden-Baden 2012, S. 27-42; Wolfgang Wessels: Das politische System der Europäischen Union, Wiesbaden 2008, S. 41-44; Wolfgang Streek: Gekaufte Zeit. Die vertagte Krise des demokratischen Kapitalismus, Berlin 2013; Beate Kohler-Koch/Thomas Conzelmann/ Michèle Knodt: Europäische Integration - Europäisches Regieren, Wiesbaden 2004; Sylvain Kahn: Histoire de la construction de l'Europe depuis 1945, Paris 2011; Robert Frank: La construction européenne une histoire cyclique, in: Questions internationales 7/2004, S. 6-21.

2 Vgl. Philip Bajon: Europapolitik „,am Abgrund“. Die Krise des „leeren Stuhls“ 1965-66, Stuttgart 2012; Philip Bajon: Die Krise des leeren Stuhls 1965/66. Verlauf und Folgen, in: Michael Gehler (Hrsg.): Vom Gemeinsamen Markt zur europäischen Unionsbildung, 50 Jahre Römische Verträge 1957-2007, Wien 2009, S. 371-392; Desmond Dinan: Governance and Institutional Developments: Ending the Constitutional Impasse, in: Journal of Common Market Studies S 1/2008, S. 71-90; Desmond Dinan: Governance and Institutions: Implementing the Lisbon Treaty in the Shadow of the Euro Crisis, in: Journal of Common Market Studies S 1/2011, S. 103-121; Robert Frank: La construction européenne une histoire cyclique, in: Questions internationales 7/2004, S. 6-21; Robert Frank: Vers un traité refondateur, in: Marie-Thérèse Bitsch (Hrsg.): Cinquante ans de traité de Rome 1957-2007, Stuttgart 2009, S. 75-95; Hartmut Kaelble: Die Krise in der Europäischen Union aus historischer Sicht, in: Zeitschrift für Staats- und Europawissenschaft 4/2006, S. 522-541; Hartmut Kaelble: Historische Krisen der europäischen Integration. Ein Essay, in: Volker Depkat/Piero S. Graglia (Hrsg.): Entscheidung für Europa Decidere 1'Europa, Berlin 2010, S. 23-35; Hartmut Kaelble: Europa in der Krise. Zivilisationskrise - Integrationskrise - Krisenmanagement, in: Thomas Mergel (Hrsg.): Krisen Verstehen. Historische und kulturwissenschaftliche Annäherungen, Frankfurt 2012, S. 131-144; Sylvain Kahn: Histoire de la construction de l'Europe depuis 1945, Paris 2011; Pieter Lagrou: La crise européenne, in: Paul Magnette/Anne Weyembergh (Hrsg.): L'Union européenne, la fin d'une crise?, Brüssel 2008, S. 15-24; Wilfried Loth (Hrsg.): Crisis and Compromises, the European project 1963-1969, Baden-Baden 2001; Piers Ludlow (Hrsg.): The European Community and the crisis of the 1960's. Negotiating the Gaullist Challenge, London 2006; Jean-Marie Palayret/Helen Wallace/Pascaline Winand (Hrsg.): Visions, votes and vetos. The empty chair crisis and the Luxemburg compromise forty years on, Brüssel 2006; Hagen Schulz-Forberg: On the historical origins of the EU's current crisis: Or, the hypocritical turn of European integration, in: Agustín José Menéndez/Edoardo Chiti/Pedro Teixeira (Hrsg.): The European Rescue of the European Union? The Existential Crisis of the European Political Project, Oslo 2012, S. 15-36; Hagen Schulz-Forberg/Bo Stråth: Soft and Strong European Public Spheres, in: Robert Frank/Hartmut Kaelble/Marie-Françoise Lévy/Luisa Passerini (Hrsg.): Building a European Public Sphere: From the 1950s to the Present, Brüssel 2010, S. 55-76; Antonio Varsori (Hrsg.): Alle origini del presente. L'Europa occidentale nella crisi degli anni settanta, Milan 2007.

3 Vgl. Richard C. Eichenberg/Russel J. Dalton: Post-Maastricht blues: The transformation of citizen support for European integration 1973-2004, in: Acta politica 2-3/2007, S. 128-152; Liesbet Hooghe/Gary Marks: A postfunctionalist theory of European integration: from permissive consensus to constraining dissensus, in: British Journal of Political Science 1/2009, S. 1-24; Dieter Fuchs/Christian Schneider: Support of the EU and European identity, in: Dieter Fuchs/Hans-Dieter Klingemann (Hrsg.): Cultural diversity, European identity and the legitimacy of the EU, Cheltenham 2011, S. 61-85; Wilfried Loth: Europäische Identität in historischer Perspektive, Zentrum für Europäische Integrationsforschung: Discussion paper C 113/2002; Wilhelm Knelangen: Europaskepsis: Die EU und der Vertrauensverlust der Bürgerinnen und Bürger, in: Aus Politik und Zeitgeschichte 4/2012, S. 32-40. 
Man kann darüber hinaus aus den zahlreichen Synthesen zur Geschichte der europäischen Integration eine Art Standardkatalog von acht Krisen der europäischen Integration herausfiltern:

- die Krise Europas nach dem Zweiten Weltkrieg 1945 bis 1951;

- die Krise nach der Ablehnung der Europäischen Verteidigungsgemeinschaft in der französischen Assemblée nationale 1954;

- die Krise des leeren Stuhls 1965/66;

- das Scheitern der Pläne zu einer Wirtschafts- und Währungsunion und der politischen Union in den frühen 1970er Jahren und die darauf folgenden ,bleiernen Jahre', eine Krise, die sich bis zur Eurosklerose der frühen 1980er Jahre hinschleppte;

- die durch die deutsche Einheit 1990 ausgelöste Krise und danach die Ratifizierungskrise des Vertrags von Maastricht 1992;

- die zeitlich parallele europäische Krise durch den jugoslawischen Bürgerkrieg 1991 bis 1995;

- die Krise des französischen und niederländischen ,Neins` zum Verfassungsvertrag ${ }^{5}$ 2005;

- schließlich die Finanz- und Schuldenkrise seit 2007.

Man kann die verschiedenen Krisen in den frühen 1990er Jahren auch als eine einzige, dann freilich sehr komplexe Krise zusammenfassen. ${ }^{6}$ Dann kommt man auf sieben Krisen der europäischen Integration.

In diesem kurzen Essay wird zuerst versucht, die bisherigen Krisen der europäischen Integration zu typisieren. Auf der Grundlage dieser Typologie werden danach die drei genannten Narrative von einer Spirale nach unten, von produktiven Krisen und von Krisenzyklen der historischen Entwicklung genauer betrachtet und zu beantworten versucht, welche dieser gegensätzlichen Vorstellungen die Geschichte der Krisen der europäischen Integration am besten abbildet.

\section{Eine Typologie der Krisen der europäischen Integration}

Die bisherigen Krisen der europäischen Integration lassen sich nicht leicht in Typen zusammenfassen. Jede Krise, auch die gegenwärtige, hat ihr eigenes Profil, ihre eigenen Kontexte und auch ihre eigene Interpretation durch die Zeitgenossen. Nur mit Abstrichen kann man deshalb eine Typologie für die bisherigen Krisen der europäischen Integration entwi-

4 Vertrag über die Europäische Union, in: Amtsblatt der EU, Nr. C 191 vom 29. Juli 1992.

5 Vertrag über eine Verfassung für Europa, in: Amtsblatt der EU, Nr. C 310 vom 16. Dezember 2004.

6 Vgl. als für diese Krisen besonders wichtige, jüngere Gesamtdarstellungen der Geschichte der europäischen Integration: Marie-Thérèse Bitsch: Histoire de la construction européenne, Paris 2004; Alasdair Blair: The European Union since 1945, Harlow 2005; Gérard Bossuat: Histoire de l'Union européenne, Paris 2009; Bernard Bruneteau: Histoire de l'unification européenne, Paris 2005; Gerhard Brunn: Die Europäsche Einigung von 1945 bis heute, 2. Auflage, Stuttgart 2009; Gabriele Clemens/Alexander Reinfeldt/Gerhard Wille: Geschichte der europäischen Integration, Stuttgart 2008; Desmond Dinan: Europe Recast. A history of the European Union, Basingstoke 2004; Jost Dülffer: Europa im Ost-West-Konflikt, München 2004; Juergen Elvert: Die europäische Integration, Darmstadt 2006; John Gillingham: European integration 1950-2003. Superstate or new market economy?, Cambridge 2003; Sylvain Kahn: Histoire de la construction de l'Europe depuis 1945, Paris 2011; Franz Knipping: Rom, 25. März 1957. Die Einigung Europas, München 2004; Wilfried Loth: Der Weg nach Europa: Geschichte der europäischen Integration 1939-1957, 3. Auflage, Göttingen 1996; Jürgen Mittag: Kleine Geschichte der Europäischen Union. Von der Europaidee bis zur Gegenwart, Münster 2008; Donato Fernández Navarrete: Historia de la Unión Europea. España como estado miembro, Madrid 2010; Bino Olivi/Allessandro Giacone: L'Europe difficile. Histoire politique de la construction européenne, 2. Auflage, Paris 2007; Hans-Joachim Seeler: Geschichte und Politik der europäischen Integration, Baden-Baden 2008; Peter Stirk: A history of European integration since 1914, London 2001; Guido Thiemeyer: Europäische Integration. Motive - Prozesse - Strukturen, Stuttgart 2010; Wessels: Das politische System der Europäischen Union, 2008. 
ckeln. Sie wird sich im Folgenden auf eine induktive Typologie beschränken, die nur die bisherigen Krisen erfassen kann und keine Idealtypen entwickeln will.

Eine solche Typologie ist ohne eine klare Definition der Krise der europäischen Integration nicht möglich. Bisher ist nicht ausgemacht, was man unter Krisen der europäischen Integration verstehen könnte. In diesem Artikel werden sie als politische Entscheidungskrisen verstanden, nicht als wirtschaftliche oder kulturelle Strukturkrisen. Sie waren Blockaden in grundlegenden politischen Entscheidungen über die europäische Integration, nicht nur in einem der vielen europäischen Politikfelder. Entscheidend ist, dass sie auch von den Zeitgenossen als Krisen wahrgenommen und nicht nur nachträglich von Historikern als Krisen eingestuft wurden. Sie stellten die Politik vor eine neue Situation und erforderten neue Konzepte für die europäische Politik. Die Krisen der europäischen Integration waren meist relativ abgeschlossene Ereignisse, begrenzt auf wenige Jahre, selten lang andauernde Krisensituationen. ${ }^{7}$

Sechs Typen von Krisen der europäischen Integration lassen sich unterscheiden. Dabei soll die Reichweite der Krise im Zentrum der Typisierung stehen. Auch die Ursachen und die Lösungen in die Typologie aufzunehmen, wäre zu komplex. Wie groß die Reichweite einer Krise war und welchem Typ sie damit angehörte, hing vor allem von drei Bedingungen ab: ob sich die Entscheidungsblockade auf die Regierungsebene der Mitgliedstaaten beschränkte oder ob auch die nationalen und europäischen Öffentlichkeiten und Zivilgesellschaften beteiligt waren; ob es sich um rein politische Krisen handelte oder ob die Krisen mit wirtschaftlichen, sozialen oder kulturellen Umbrüchen verschränkt waren; ob sich die Krise auf Europa beschränkte oder ob auch außereuropäische Mächte und Öffentlichkeiten an den Konflikten beteiligt waren. Um Missverständnisse zu vermeiden, sei betont, dass mit der folgenden Typologie in keiner Weise eine historische Entwicklung suggeriert werden soll. Die Typologie beschränkt sich zudem auf die Geschichte der europäischen Integration seit den 1950er Jahren. Eine Typologie der Krisen des internationalen europäischen Systems seit dem Mittelalter wäre reizvoll, würde aber zum Verständnis der heutigen Krise wohl nicht mehr beitragen und könnte nur von einer Gruppe von Historikern mit unterschiedlicher Expertise entwickelt werden.

\section{Erster Krisentyp: Krisenmanagement}

Ein erster Typ von Krise der europäischen Integration ist das bloße Krisenmanagement. Es handelt sich um lokale Krisen, die innerhalb oder auch außerhalb des Territoriums der Europäischen Union entstehen und die ein Krisenmanagement der Europäischen Union verlangen. Normalerweise entstehen daraus keine Krisen der europäischen Integration. Dies geschieht nur dann, wenn die Europäische Union in eine Entscheidungsblockade gerät, sei es, weil die Mitgliedstaaten in einen unlösbaren Konflikt miteinander gerieten, sei es, weil die Europäische Union keine Kompetenzen für die Lösung dieses Konflikts besitzt.

Ein Beispiel für ein Krisenmanagement, das nicht zu einer Krise der Europäischen Union führte und deshalb auch nicht in der Liste der Krisen der europäischen Integration auftaucht,

7 Das brillante und viel verwandte Krisenkonzept von Reinhart Koselleck, der Krisen vor allem als Folge von öffentlicher Kritik sah, lässt sich auf Europa als Ganzes nur schwer anwenden, da sich die europäische Ö̈fentlichkeit und damit auch die Kritik an der europäischen Politik schwächer entwickelte als in den europäischen Staaten seit dem 18. Jahrhundert, teils weil die meisten Intellektuellen nur im Rahmen ihrer jeweiligen nationalen Öffentlichkeit handelten, teils aber auch, weil nicht so klar war, wer der Adressat der europäischen öffentlichen Kritik hätte sein können, da das machtvollste Entscheidungszentrum der Europäischen Union, der Europäische Rat, aus den nationalen Regierungschefs besteht, die sich in der Regel nach den Entscheidungen des Europäischen Rates wieder an ihre nationalen Öffentlichkeiten wenden. 
ist der militärische Konflikt zwischen Georgien und Russland 2008, in dem der Präsident des Europäischen Rates intervenierte und eine Lösung zur Beendigung der militärischen Auseinandersetzungen fand. Ein umgekehrtes Beispiel für ein Krisenmanagement, das zu einer Krise der europäischen Integration eskalierte, war der Bürgerkrieg in Jugoslawien 1991 bis 1995. Die europäische Öffentlichkeit erwartete, dass die Europäische Union als Akteur bei der Lösung dieser Krise auftreten würde. Der Jugoslawienkrieg traf den Nerv der europäischen Identität, weil Friedenssicherung in Europa ein zentraler Bestandteil der europäischen Identität ist und erstmals seit dem Zweiten Weltkrieg wieder ein Krieg auf europäischem Boden stattfand. Da die Europäische Union keine militärische Eingreiftruppe besitzt, war sie aber nicht in der Lage, diese Krise zu managen und scheiterte. Die Jugoslawienkrise wurde deshalb auch in der Öffentlichkeit als Krise der europäischen Integration wahrgenommen.

\section{Zweiter Krisentyp: diplomatische Entscheidungsblockade}

Ein zweiter Typ von Krise ist die diplomatische Entscheidungsblockade. Diese Krise besteht aus grundsätzlichen Konflikten über die europäische Integration, beschränkt sich aber auf die Regierungen der Mitgliedstaaten und ist deshalb eine rein diplomatische Angelegenheit ohne eine massive Beteiligung der Medien, der Öffentlichkeit und der Zivilgesellschaft. Sie ist auch nicht begleitet von wirtschaftlichen oder kulturellen Umbrüchen und bleibt zudem begrenzt auf die Mitgliedstaaten der Europäischen Gemeinschaft beziehungsweise der Europäischen Union. Allerdings wird sie von den beteiligten Regierungen als eine schwerwiegende Krise wahrgenommen.

Musterbeispiel dafür ist die Krise des leeren Stuhls 1965/1966, ein grundlegender Konflikt zwischen den Mitgliedsregierungen über die Entscheidungsformen der Europäischen Wirtschaftsgemeinschaft, der sich schon mit dem einseitigen Veto Charles de Gaulles gegen den Beitritt Großbritanniens zur Europäischen Wirtschaftsgemeinschaft 1963 aufbaute und durch den einseitigen Austritt Frankreichs aus den militärischen Strukturen der NATO ${ }^{8}$ noch verschärft wurde. Die französische Regierung unter de Gaulle lehnte 1965/66 die vertraglich vorgesehenen Mehrheitsentscheidungen ab und arbeitete in den europäischen Gremien nicht mehr mit, da die anderen Regierungen Mehrheitsentscheidungen unterstützten. Dieser grundsätzliche Konflikt über das Procedere der Entscheidungen wurde von den Regierungen auch als Krise wahrgenommen.

\section{Dritter Krisentyp: Konflikte zwischen Regierungen und mobilisierter Öffentlichkeit}

Ein dritter Typ der Krise besteht aus Konflikten um grundlegende europäische Entscheidungen zwischen Regierungen und mobilisierter Öffentlichkeit. In diesem Typ von Krise entstehen Entscheidungsblockaden nicht allein zwischen den europäischen Regierungen der Mitgliedstaaten. Auch die Öffentlichkeit, die Medien, die Experten und die Zivilgesellschaft stehen im Dissens über grundlegende europäische Entscheidungen. Dieser Krisentyp ist ebenfalls rein politisch und nicht von einem wirtschaftlichen oder kulturellen Umbruch begleitet. Er ist auch auf Europa beschränkt. Außereuropäische Akteure spielen keine wesentliche Rolle. Aber er ist doch mehr als eine rein diplomatische Angelegenheit. Er mobilisiert auch die Bürger und die Medien.

8 North Atlantic Treaty Organization. 
Ein erstes Beispiel ist die Krise um das ,Nein“ der Assemblée nationale 1954 zur Europäischen Verteidigungsgemeinschaft, mit der eine stark integrierte europäische Armee und zudem eine europäische Politische Union von den sechs Regierungen der Montanunion geschaffen werden sollten. Dieses ,Nein' war nur der Endpunkt einer Krise, in der gegensätzliche Vorstellungen der beteiligten Regierungen über die grundlegende Frage der militärischen Integration Europas im Kalten Krieg und während des französischen Indochinakriegs einander gegenüberstanden. Der Vertrag der Europäischen Verteidigungsgemeinschaft war ein schwieriger Kompromiss, wurde von mehreren Regierungen nur halbherzig unterstützt und scheiterte am Ende an der dilatorischen Behandlung durch die französische Regierung in der Assemblée nationale. Die Öffentlichkeit war besonders in Frankreich stark aufgerührt und tief gespalten.

Ein weiteres Beispiel für diesen Typ von Krise ist der Konflikt, der in den europäischen Institutionen durch die deutsche Einheit ausgelöst wurde. Es entstand ein grundlegender Dissens über die deutsche Einheit und über die europäische Einbindung des vereinten Deutschlands zwischen den beteiligten europäischen Regierungen. Bundeskanzler Helmut Kohl legte ein Zehn-Punkte-Programm zur deutschen Einheit vor, das mit anderen Mitgliedstaaten nicht abgesprochen war. Zwar war die Europäische Kommission unter Jacques Delors rasch bereit, die DDR als Teil Deutschlands in die Europäische Gemeinschaft aufzunehmen. Der damalige französische Präsident François Mitterrand hingegen stand der deutschen Einheit vorübergehend, die damalige englische Premierministerin Margaret Thatcher dauerhaft skeptisch gegenüber. Auch die Medienöffentlichkeiten in Europa waren über die deutsche Einheit und die Einbindung Deutschlands in die Europäische Gemeinschaft tief gespalten und stark mobilisiert. Ganz passt diese Krise nicht zu diesem Typ, weil die deutsche Einheit vor allem von den USA und der UdSSR entschieden wurde. Aber den Beschluss über die aus der deutschen Einheit folgende Weiterentwicklung der europäischen Integration, also der Vertrag von Maastricht, wurde allein von europäischen Regierungen gefällt. Zu dieser Krise gehörte auch, dass die Ratifizierung des Vertrags von Maastricht in Gefahr geriet, weil er in einem Referendum in Dänemark abgelehnt wurde und in Großbritannien ein Referendum mit negativem Ausgang drohte. Auch an diesem Akt der Krise waren Regierungen, aber ebenso die Öffentlichkeit in Dänemark und Großbritannien massiv beteiligt.

Ein drittes, wiederum etwas anders gelagertes Beispiel dieses Typs von Krise ist die Ablehnung des Verfassungsvertrags 2005 in den französischen und niederländischen Referenden. Dieser Verfassungsvertrag kam zwar am Ende als ein Kompromiss zwischen den beteiligten 15 Regierungen nach jahrelangen Verhandlungen und nach intensiver Beratung im Europäischen Konvent zustande. Die nationalen Öffentlichkeiten waren aber über den Verfassungsvertrag tief gespalten. In den Meinungsumfragen zeichnete sich in den meisten Mitgliedstaaten keine klare Mehrheit ab. Das knappe Ergebnis des Referendums in Frankreich spiegelte diese tiefe Spaltung der europäischen Öffentlichkeit wider.

\section{Vierter Krisentyp: wirtschaftliche und kulturelle Umbrüche}

Ein vierter Typ der Krise besteht nicht nur aus Entscheidungsblockaden zwischen den Regierungen der Mitgliedstaaten und tief gespaltenen Öffentlichkeiten, sondern ist begleitet und beeinflusst durch gleichzeitige wirtschaftliche und kulturelle Umbrüche. Auch in der öffentlichen Wahrnehmung begrenzen sich Krisen dieses Typs nicht auf die europäische Integration, sondern werden als umfassendere europäische Krisen wahrgenommen. Die Re- 
gierungen und die Öffentlichkeiten stehen vor einer neuen Situation und sind gezwungen, ihr Selbstverständnis und ihre politischen Konzepte grundlegend zu ändern.

Ein Beispiel für diese Krise waren die frühen 1970er Jahre, als nicht nur die hochfliegenden europäischen Pläne zur Wirtschafts- und Währungsunion beziehungsweise zur politischen Union scheiterten und die Norderweiterung der Europäischen Gemeinschaft von Norwegen abgelehnt und von Großbritannien mit großer Skepsis betrachtet wurde. Gleichzeitig erlebte Europa einen tiefen wirtschaftlichen Umbruch, das Ende des Währungssystems von Bretton Woods, die Ölkrise von 1973, das rapide und dauerhafte Absinken des wirtschaftlichen Wachstums und später die starke Inflation. Auch die Mentalitäten veränderten sich. Der Zukunfts- und Planungsoptimismus der 1950er und 1960er Jahre lief aus und wurde durch skeptische Zukunftserwartungen verdrängt. Diese neuen wirtschaftlichen und kulturellen Bedingungen änderten auch die europäische Integration grundlegend. Ein langsamer Prozess des Suchens nach Lösungen für die gescheiterte Wirtschafts- und Währungsunion und für die weitgehend gescheiterte politische Union begann. Anders als die anderen Krisen der europäischen Integration dauerte diese Krise besonders lange, schien in den späten 1970er Jahren mit neuen Integrationsschritten wie den Direktwahlen zum Europäischen Parlament und dem Europäischen Währungssystem beendet zu sein, brach allerdings in den frühen 1980er Jahren mit der Eurosklerose, dem Zurückfallen der europäischen Wirtschaft hinter die anderen westlichen Wirtschaften und neuen Anforderungen an die europäische Integration wieder auf. Diese Krise war auch begleitet von einer starken Skepsis gegenüber der europäischen Integration in der öffentlichen Diskussion.

\section{Fünfter Krisentyp: globale Entscheidungskrise}

In einem fünften Typ der Krise kommt eine entscheidende geografische Dimension hinzu: die globale Entscheidungskrise. Dieser Typ von Krise beschränkt sich nicht auf Europa und spaltet nicht nur die Regierungen und die Öffentlichkeiten der Mitgliedstaaten. Auch die Regierungen und Öffentlichkeiten außereuropäischer Staaten sind von dieser Krise betroffen, oft auch durch sie gespalten. Sie versuchen deshalb Einfluss auf die europäischen Entscheidungen und auf die europäischen Öffentlichkeiten zu nehmen. Vor allem wenn außereuropäische Großmächte beteiligt sind, verändert sich der Charakter der Krise auch für Europäer. Neue Wahrnehmungen einer globalen gemeinsamen Aufgabe, aber auch Bilder einer von außen auferlegten und aufgedrängten Krise können in diesem Typ von Krise entstehen.

Die Krise seit 2007 entspricht diesem Typ weitgehend. Sie wurde angestoßen durch die internationale, von den USA ausgehende Finanzkrise. Diese Finanzkrise löste in dem ohne die erforderlichen Wirtschafts- und Finanzkompetenzen konstruierten europäischen Währungssystem eine europäische Schuldenkrise, extrem hohe Zinsen für Staatsanleihen der europäischen Südstaaten und eine wachsende Skepsis gegenüber dem europäischen Währungssystem aus und belastete nicht nur die europäischen, sondern auch außereuropäische Wirtschaften. Sie zwang zu Vorbereitungen für weitere Integrationsschritte, für eine stärkere europäische Kontrolle der nationalen Staatshaushalte und des europäischen Bankensystems. Die Krise wird auch in der europäischen Medien- und Expertenöffentlichkeit als eine besonders schwere Krise wahrgenommen. Da von dieser Schuldenkrise und ihrer Lösung durch die Europäische Union auch die USA, China, Indien und viele andere Staaten betroffen sind, versuchen nicht nur die Regierungen der Großmächte, sondern auch der Internationale Währungsfond, die Gruppe der G20 und internationale Experten Einfluss auf die europäischen Entscheidungen zu nehmen. 


\section{Sechster Krisentyp: Zivilisationskrise}

Eine sechste Krise ist die Zivilisationskrise, die schwerste Variante der Krisen der europäischen Integration. Sie ist nicht allein eine Entscheidungsblockade der europäischen Regierungen, auch nicht allein eine tiefe Spaltung der Medien- und Expertenöffentlichkeit in Europa, ist auch nicht nur verbunden mit einem wirtschaftlichen oder kulturellen Umbruch. Sie ist vielmehr eine fundamentale Krise des gesamten wirtschaftlichen und politischen Systems, ein tiefgehender, gesellschaftlicher und kultureller Verfall mit der Gefahr der Auflösung der politischen und gesellschaftlichen Werte und überhaupt des Bestandes Europas. Auch diese Krise wird von der Öffentlichkeit in ihrem ganzen Ausmaß wahrgenommen. In einer solchen Krise wird eine Zivilisation normalerweise durch außenstehende Mächte unterworfen oder zumindest in ihren Einflussbereich eingeordnet.

Das einzige Beispiel für eine solche Krise in der neueren Geschichte Europas sind die Jahre unmittelbar nach dem Ende des Zweiten Weltkrieges, in denen Millionen von Familien Tote und Invaliden zu beklagen hatten, viele europäische Städte und Verkehrswege zerstört waren, Millionen von Europäern ohne Hoffnung wortwörtlich auf der Straße lagen, viele Europäer ohne Einkommen, ohne Essen, ohne Wohnung waren, Europa in einem tiefen Pessimismus versank, aufgeteilt und dem sowjetischen Imperium beziehungsweise der Vormacht der USA zugeordnet wurde. Diese europäische Zivilisationskrise war gleichzeitig auch eine Krise der europäischen Integration, da sie zwar viele Ideen und mehrere, gleich noch zu besprechende, Anläufe zur europäischen Integration hervorbrachte, die aber meist rasch wieder in eine Krise gerieten oder als randständige Organisationen der europäischen Integration endeten.

\section{Welche Geschichte: Immer schärfere Krisen oder Krisenzyklen oder produktive Krisen?}

Nach diesem typisierenden Überblick über die Krisen der europäischen Integration kann die Frage besser beantwortet werden, welche der drei anfangs erwähnten Vorstellungen die Entwicklung der vergangenen rund 60 Jahre am besten fasst: die Katastrophenvorstellung, die die immer tieferen Krisen demnächst in dem Zusammenbruch der europäischen Integration enden sieht oder die Vorstellung von Krisenzyklen, in denen jeweils neue Generationen die Krise der Europapolitik der älteren Generation erleben und eine neue Europapolitik entwickeln oder die Vorstellung von der produktiven Krise, die einen neuen Schritt in der europäischen Integration auslöst, freilich auch alle Ängste und Zusammenbruchsfantasien einer Krise braucht, um den politischen Druck für die nächsten Integrationsschritte aufzubauen.

Für das Katastrophenszenario sprechen die bisherigen Krisen der europäischen Integration nicht. Die schwerste Krise Europas stand nicht am angeblichen Ende, sondern am Anfang der Geschichte der europäischen Integration: die Krise direkt nach dem Zweiten Weltkrieg, die soeben angesprochen wurde. Selbst diese Krise war jedoch kein Todesstoß. Es ist vielmehr erstaunlich, dass in dieser tiefen materiellen und moralischen Depression Politiker und Intellektuelle die Initiative zur Gründung von europäischen Institutionen ergriffen. Vier Anläufe zur europäischen Integration wurden gemacht, von denen die meisten allerdings rasch in eine Krise gerieten oder die Erwartungen nicht erfüllten. Die Nachkriegszeit war daher eine Mischung aus Krise und Aufbruch der europäischen Integration: 1948 wurde die 
OEEC, ${ }^{9}$ eine unter dem Druck der USA als rein wirtschaftliche europäische Organisation für West- und Osteuropa gegründet. Sie wurde allerdings von der UdSSR nach Verhandlungen für ihre gesamte Einflusszone abgelehnt und traf auch bei den westeuropäischen Regierungen auf starkes Misstrauen, war eine bloße Koordinationsinstanz für die Durchführung des Marshallplans und wurde 1961 in eine politische Beratungsorganisation unter dem heutigen Namen OECD ${ }^{10}$ umgewandelt. Ebenfalls 1948 wurde die Westeuropäische Union als eine militärische Integration während des Kalten Krieges zum Schutz vor einer erneuten deutschen Aggression von fünf westeuropäischen Staaten gegründet, versank allerdings mit der Gründung der atlantischen NATO 1949 schon bald in der Bedeutungslosigkeit. 1949 wurde der Europarat gegründet, der unter der Führung Großbritanniens einen Großteil der westeuropäischen Länder unter voller Wahrung ihrer nationalen Souveränität zusammenschloss, politische und kulturelle Ziele besaß und vor allem den Straßburger Menschenrechtsgerichthof einrichtete, daneben aber auch berühmte europäische Kunstausstellungen organisierte. Der Europarat erfüllte allerdings die Erwartungen in wichtigen kontinentaleuropäischen Staaten an eine handlungsfähige, wirtschaftliche und politische, europäische Entscheidungsinstanz nicht. 1951 wurde deshalb schließlich die Montanunion gegründet, die unter der Initiative Frankreichs den Kohlebergbau und die Eisen- und Stahlindustrie von sechs kontinentaleuropäischen Staaten in einer supranationalen Organisation zusammenschloss und mit der wechselseitigen Kontrolle der damals wichtigsten Rüstungsindustrien den europäischen Frieden sichern und mehr Wohlstand schaffen wollte. Erst in diesem vierten Anlauf wurde am Ende der Nachkriegskrise die Ausgangsorganisation für die heutige Europäische Union geschaffen.

Auch die Krise während der frühen 1970er Jahre war schwer, auch wenn sie einen ganz anderen Charakter besaß als die heutige Krise. Sie war nicht nur eine wirtschaftliche und kulturelle Krise mit dem Abbruch des internationalen Währungssystems von Bretton Woods durch die amerikanische Regierung, mit dem dramatischen Anstieg der Ölpreise durch die Ölkrisen, mit dem drastischen Rückgang der hohen wirtschaftlichen Wachstumsraten, mit unerträglich hohen Inflationsraten und mit einem starken Rückgang des Zukunfts- und Planungsoptimismus. Sie war auch eine schwere Krise der europäischen Integration, eine tiefe Enttäuschung nach dem Scheitern der hochfliegenden Pläne zum Aufbau einer europäischen politischen Union, einer Wirtschafts- und Währungsunion und angesichts der Schwierigkeiten der Norderweiterung nach dem norwegischen ,Nein' und der konfliktreichen britischen Mitgliedschaft. Diese Krise war so schwer, dass erst wieder 1979 in ersten, kleinen, nicht von großem Erfolg begleiteten Schritten mit dem Europäischen Währungssystem (EWS) und mit den Direktwahlen zum Europäischen Parlament neue Integrationsschritte unternommen und erst Mitte der 1980er Jahre mit der Einheitlichen Europäischen Akte ${ }^{11}$ und dem Binnenmarkt der europäischen Integration wieder entscheidenden Auftrieb gegeben werden konnte. Angesichts der Schwere der Krise der Nachkriegszeit und der Krise der 1970er Jahre erscheint die gegenwärtige Krise nicht mehr als so einzigartig tief. Das Konzept einer kontinuierlichen Spirale nach unten in immer schwerere Krisen der europäischen Integration überzeugt daher nicht.

Fasst die Vorstellung von Zyklen die Geschichte der Krisen der europäischen Integration besser? Auch als Zyklen lassen sich die Krisen der europäischen Integration allerdings nur

9 Organisation for European Economic Co-operation.

10 Organisation for Economic Co-operation and Development.

11 Einheitliche Europäische Akte, in: Amtsblatt der EU, Nr. L 169 vom 29. Juni 1987. 
schwer einordnen. Die Geschichte der Krisen der europäischen Integration ist zu kurz. Es wäre voreilig, aus den drei schweren Krisen schon auf einen Zyklus schließen zu wollen. Die bisherigen schweren Krisen besaßen darüber hinaus zu wenig Ähnlichkeit, als dass man sie wirklich als Zyklen ansehen könnte. Die erste dieser drei Krisen war völlig durch die Folgen des Zweiten Weltkriegs bestimmt, während die anderen Krisen mit Kriegen nichts zu tun hatten. Die Krise der 1970er Jahre bestand in einem Scheitern von neuen europäischen Projekten. Die dritte, gegenwärtige, schwere Krise hingegen ist im Kern eine Verteidigungskrise des Euro. Darüber hinaus lassen sich diese Krisen nicht wirklich als Ablösung einer älteren durch eine jüngere Generation in der Europapolitik interpretieren. In der Krise nach dem Zweiten Weltkrieg waren die Gründungsväter durchweg alte Männer, die das Europaprojekt eines Gleichaltrigen, Hitlers, und dessen Projekt eines durch das NS-Deutschland beherrschten und ausgebeuteten Europas revidierten. In den 1970er Jahren scheiterten dagegen nicht Projekte dieser alten Männer, sondern umgekehrt die Projekte einer neuen Generation von Persönlichkeiten wie Willy Brandt, Georges Pompidou, Leo Tindemans und Pierre Werner. Man kann die heutige Krise als das fatale Erbe der schlecht konstruierten Wirtschafts- und Währungsunion der Generation Helmut Kohl und François Mitterrand ansehen, aber bisher ist nicht klar genug erkennbar, ob wirklich eine jüngere Generation von Europapolitikern mit einem neuen Konzept der europäischen Einheit auftritt und sich auch durchsetzt.

Dagegen fasst die dritte Vorstellung von den produktiven europäischen Krisen, in denen die europäische Integration regelmäßig weiter vorangetrieben wird, die historische Wirklichkeit noch am ehesten, wenn auch nicht voll. Drei Kritikpunkte werden gegen dieses Konzept angeführt.

Krisen wurden zwar beendet, aber nicht immer wirklich gelöst. Zwei Beispiele von solchen nicht produktiven Krisen seien erwähnt. Die Krise des ,Nein' der französischen Assemblée nationale 1954 zu dem Projekt der Europäischen Verteidigungsgemeinschaft wurde nach manchen Lehrbüchern schon 1957 durch die Gründung der Europäischen Wirtschaftsgemeinschaft gelöst. Tatsächlich scheiterte 1954 die europäische militärische Integration auf Dauer. Bis heute gibt es keine integrierte europäische Armee. Stattdessen wurden die europäischen Armeen unter der Führung der USA in der atlantischen Integration der NATO zusammengeführt. Die Europapolitik wurde in der Krise von 1954 zu einem Strategiewechsel gezwungen und setzte für die nächsten Jahrzehnte vor allem auf wirtschaftliche statt auf militärische und politische Integration. Ähnlich ungelöst blieb auch, wie immer wieder herausgestrichen wird, die Krise des leeren Stuhls 1965/66. Sie wurde zwar durch den Luxemburger Kompromiss beendet. Aber dieser umstrittene Kompromiss war nicht produktiv, sondern schloss für längere Zeit Mehrheitsentscheidungen unter den europäischen Regierungen aus und hielt damit an schwerfälligen Entscheidungsprozeduren fest.

An der Vorstellung von produktiven Krisen der europäischen Integration wird zudem oft zu Recht kritisiert, dass die scheinbar produktiven Lösungen der Krisen oft Defizite enthielten, die zur Ursache für die nächste Krise wurden. ${ }^{12}$ In der gegenwärtigen Krise wird be-

12 Vgl. historische Arbeiten zu im Folgenden nicht behandelten Strukturdefiziten, die zu Krisen führten: Antonio Varsori: Les défis de 1'Europe sociale, in: Marie-Thérèse Bitsch (Hrsg.): Cinquante ans de traité de Rome 1957-2007, Stuttgart 2009, S. 185-201; Bo Stråth: Die enttäuschte Hoffnung auf das soziale Europa, in: Arnd Bauerkämper/Hartmut Kaelble (Hrsg.): Gesellschaft in der europäischen Integration seit den 1950er Jahren. Migration - Konsum - Sozialpolitik - Repräsentationen, Stuttgart 2012, S. 23-42; Max Haller: Die europäische Integration als Elitenprozess. Das Ende eines Traums?, Wiesbaden 2009; Simon Hix: What's Wrong with the European Union and How to Fix It, Cambridge 2008; Guido Thiemeyer: Die Ursachen des Demokratiedefi- 
sonders häufig das folgende Beispiel zitiert: Die Krise, die 1990 durch die deutsche Einheit ausgelöst wurde, fand ihre Lösung im Vertrag von Maastricht und dort vor allem in der noch stärkeren Einbindung Deutschlands in die europäische Wirtschaft durch die Schaffung der gemeinsamen Währung. Diese Lösung enthielt jedoch ein schwerwiegendes Defizit: Der neuen Währung fehlte das Fundament einer Finanz- und Wirtschaftsunion, vor allem die europäische Kontrolle der nationalen Staatshaushalte und Banken. Dieses Defizit wurde zu einer entscheidenden Ursache für die gegenwärtige Schuldenkrise. ${ }^{13}$ Ein anderes Beispiel ist die Krise, die nach dem weitgehenden Scheitern der politischen Union während der frühen 1970er Jahre folgte. Sie wurde in mehreren kleinen Schritten zu lösen versucht. Zu diesen Schritten gehörte auch die erste Direktwahl zum Europäischen Parlament 1979. Diese Direktwahl enthielt allerdings ein schweres Defizit: Das Europäische Parlament konnte anders als die meisten nationalen Parlamente keine Regierung wählen. Deshalb hatten die europäischen Wähler in diesen Wahlen auch keine klare Auswahl zwischen unterschiedlichen Regierungsmannschaften. Die Wahlbeteiligung nahm daher in den folgenden Jahren immer mehr ab. Europäische Politik und die europäischen Wähler entfremdeten sich einander. Diese Entfremdung war ein wichtiger Grund für die Ratifizierungskrise des Vertrags von Maastricht 1992 und für die Ablehnungskrise des Verfassungsvertrags 2005. ${ }^{14}$

Das Konzept der produktiven Krise besitzt noch eine dritte Schwäche: Es wird damit nicht selten postuliert, dass die Krise als solche zu einem Wandel der politischen Strategien und zu weiteren Schritten der europäischen Integration geführt hat. Diese Schwäche hat vielleicht mit einer traditionsreichen europäischen Denkfigur, dem Denken in Krise und Läuterung zu tun. Dabei wird jedoch übersehen, dass für einen produktiven Ausgang der Krisen in der bisherigen Geschichte der europäischen Integration immer bestimmte Voraussetzungen gegeben waren, ohne die die Krisenlösungen nicht gefunden worden wären.

\section{Fünf dauerhafte Voraussetzungen für den produktiven Ausgang von Krisen}

Es gab wenigstens fünf dauerhafte Voraussetzungen, die als eine Art Kontinuitäten in den europäischen Krisen der letzten rund 60 Jahre angesehen werden können, auch wenn sie in

zits der Europäischen Union aus geschichtswissenschaftlicher Perspektive, in: Wilfried Loth (Hrsg.): Das europäische Projekt zu Beginn des 21. Jahrhunderts, Opladen 2001, S. 27-47.

13 Vgl. dazu etwa Henrik Enderlein: Krise in der Eurozone, in: Aus Politik und Zeitgeschichte 42/2010, S. 7-12; Jens Beckert/Wolfgang Streek: Die Fiskalkrise und die Einheit Europas, in: Aus Politik und Zeitgeschichte 44/2012, S. 7-17; Werner Abelshauser: It's not the economy, stupid. Die politische Ökonomie der europäischen Integration in der Krise, in: Zeitschrift für Staats- und Europawissenschaften 1/2010, S. 1-23; Werner Abelshauser: Die Erblast des Euro - eine kurze Geschichte der Europäischen Währungsunion, in: Aus Politik und Zeitgeschichte 43/2010, S. 39-45; Ulrich Lappenküper: Mitterrand und Deutschland. Die enträtselte Sphinx, Quellen und Darstellungen zur Zeitgeschichte, Band 89, München 2011; Tilo Schabert: Wie Weltgeschichte gemacht wird - Frankreich und die deutsche Einheit, Stuttgart 2002.

14 Vgl. zur breiteren Forschung über den Euroskeptizismus: Jeffrey T. Checkel/Peter J. Katzenstein (Hrsg.): European identity, Cambridge 2009; Eichenberg/Dalton: Post-Maastricht blues, 2007; Dieter Fuchs et al. (Hrsg.): Euroscepticism. Images of Europe among mass publics and political elites, Opladen 2009; Viktoria Kaina: Wir in Europa. Kollektive Identität und Demokratie in der Europäischen Union, Wiesbaden 2009; Wilhelm Knelangen: Die EU und der Vertrauensverlust der Bürgerinnen und Bürger, in: Aus Politik und Zeitgeschichte 4/2012, S. 32-40; Aleks Szczerbiak/Paul Adam Taggart: Opposing Europe? The comparative politics of euroscepiticism, Oxford 2008; Simone Weske: Europapolitik im Widerspruch. Die Kluft zwischen Regierenden und Regierten, Wiesbaden 2011; Bernhard Weßels: Spielarten des Euroskeptizismus, in: Frank Decker/Markus Höreth (Hrsg.): Die Verfassung Europas. Perspektiven des Integrationsprojekts, Wiesbaden 2009; Sabine Voglrieder: Europäische Identität und Europäische Union: Das Selbst- und Europaverständnis der EU im Kontext der Vertiefungs-/ Erweiterungsdebatte, in: Wilfried Loth (Hrsg.): Das europäische Projekt zu Beginn des 21. Jahrhunderts, Opladen 2001, S. 175-202. 
jeder Krise etwas anders wirkten und in der gegenwärtigen Krise auf eine besonders harte Probe gestellt werden.

(1) Die europäische Integration wurde von einer Mehrheit der europäischen Bürger durchgehend als ein positives Projekt angesehen. Die Unterstützung schwankte zwar von Mitgliedstaat zu Mitgliedstaat stark und veränderte sich auch erheblich während der Geschichte der europäischen Integration, teils durch die Politisierung der Europäischen Union und durch das Ende des erwähnten permissiven Konsensus, teils auch durch die Krisen. Aber auch in Krisenzeiten blieben die Unterstützer der Europäischen Union als Ganzes immer zahlreicher als die Gegner, in der gegenwärtigen Krise ist die Mehrheit der Befürworter allerdings sehr knapp. ${ }^{15}$ Die Erwartungen an die Europäische Union blieben selbst in Krisenzeiten und in Staaten mit großer Skepsis immer hoch. Auch in der derzeitigen Krise erwartet eine große Mehrheit der Europäer vor allem in der Außen- und Sicherheitspolitik, in der Umwelt-, Antiterrorismus- und Einwanderungspolitik, mehrheitlich auch in der Wirtschafts- und Währungspolitik viel von der Europäischen Union und in der Regel mehr als von den Nationalstaaten. ${ }^{16}$

(2) Darüber hinaus wurde die europäische Integration von der großen Mehrheit der politischen Eliten, der Politiker, der Spitzenbeamten, der Experten, der Journalisten und der Intellektuellen, der Interessenverbände und Zivilgesellschaften unterstützt, wenn man von der oft rituellen Kritik an einzelnen Politiken der Europäischen Union absieht. Die Kreise der politischen Eliten, die an der Vorbereitung oder Ausführung von europäischen Entscheidungen, an der Kompromissfindung, in den europäischen Verwaltungen oder an der öffentlichen Diskussion über Europa beteiligt waren, erweiterten sich in den letzten Jahrzehnten immer mehr. Die internationale Elitenvernetzung im Bereich der europäischen Integration nahm zu. Diese Elitenvernetzung trug viel zur Überbrückung der Interessengegensätze innerhalb Europas bei, auch in der gegenwärtigen Verschärfung der Nord-Süd-Gegensätze.

(3) Die lange eingespielte französisch-deutsche Zusammenarbeit war für die Entscheidungen in den Krisen der europäischen Integration meist von großem Gewicht. Sie hat sich zwar im Laufe der sechs Jahrzehnte europäischer Integration stark gewandelt, war zeitweise offener, zeitweise exklusiver, erlebte Zeiten der Spannungen und Zeiten der Rückkehr zur Zusammenarbeit. Ihre Stärke lag nicht nur in der Größe der beiden Staaten und den fehlenden politischen Alternativen zu dieser bilateralen Zusammenarbeit und Führung. Zwischen Frankreich und Deutschland mussten zudem oft Kompromisse zu weit auseinander liegenden Interessen gefunden werden. Genau in solchen schwierigen Kompromissen konnten sich andere Mitgliedsländer oft wiederfinden.

(4) Die dauerhafte Anziehungskraft der Europäischen Union als Aktionsfeld für nationale Politiker, auch für nationale Experten und Intellektuelle, beruhte nicht allein auf den Geldern, die die Union umverteilte. Sie gründet auch auf dem globalen politischen Einfluss der Europäischen Union, an der die nationalen Politiker teilhaben konnten, sowie auf den Chancen zur Lösung von Problemen, die auf der nationalen Ebene etwa in den Bereichen der Sicherheit, der Umwelt, der Energie, der Kriminalität, der Migration, des Konsumentenschutzes oder der Bildungsstandards kaum lösbar waren. Weitere Gründe für die Anziehungskraft

15 Europäische Kommission: Standard Eurobarometer 79. Frühling 2013. Die Öffentliche Meinung in der Europäischen Union. Erste Ergebnisse, Befragung: Mai 2013, Veröffentlichung: Juli 2013, S. 10 und Annex Tabelle 46.

16 Letzte Umfrage dazu: Europäische Kommission: Standard Eurobarometer 75. Die Öffentliche Meinung in der Europäischen Union. Die wirtschaftliche Lage aus der Sicht der europäischen Bürger, Befragung: Mai 2011, Veröffentlichung: August 2011, S. 67-77. 
sind die Furcht vor unübersehbaren und gefährlichen Folgen einer Auflösung oder Entmachtung der Europäischen Union und schließlich auch die Möglichkeit, falsche politische Entscheidungen der eigenen Regierung der Europäischen Union anlasten zu können.

(5) Schließlich konnte die Europäische Union trotz aller Mängel und Fehlentscheidungen gegenüber den Bürgern und den Eliten auf eine ganze Reihe von Leistungen verweisen. Sie trieb nicht nur die wirtschaftliche Verflechtung zwischen den Mitgliedstaaten voran, ohne die ein Rückfall in die wirtschaftliche Misere der Zwischenkriegszeit drohte. Sie konnte auch in soeben erwähnten internationalen Themenfeldern mehr erreichen als ein mittelgroßer oder kleiner europäischer Staat. Sie sicherte den Europäern einen globalen Einfluss, ohne in kostenträchtige und viele Menschenleben fordernde imperiale Projekte zurück zu verfallen. Sie war auch die einzige Institution, die die Demokratie und den Frieden in Europa zu sichern vermochte.

In der durch diese fünf Voraussetzungen und damit auch Einschränkungen qualifizierten Form erfasst das Konzept der produktiven Krise die Geschichte der Krisen der europäischen Integration noch am ehesten, besser jedenfalls als die anderen Konzepte. Allerdings wissen wir nicht, ob dieses für die Vergangenheit recht wirklichkeitsnahe Konzept auch für die Zukunft der europäischen Integration gelten wird.

\section{Zusammenfassung}

Insgesamt brach in der bisherigen Geschichte der europäischen Integration seit den 1950er Jahren eine überraschende Vielfalt von Krisen aus. Jede der acht Krisen verlief anders. Dieser Artikel enthält einerseits einen Vorschlag zur Typisierung dieser Vielfalt von Krisen von dem blockierten Management lokaler Krisen bis zur fundamentalen Zivilisationskrise und erlaubt damit eine bessere Einordnung dieser Krisen. Er überprüft andererseits, wie nahe drei verbreitete Vorstellungen von der Geschichte der Krisen der europäischen Integration an die historische Wirklichkeit herankommen:

- die Vorstellung von einer Spirale nach unten in immer schwerer werdenden Krisen, die demnächst in einem Zusammenbruch der europäischen Integration enden könnten;

- die Vorstellung von einem Zyklus von Krisen, in denen die Europapolitik einer älteren Generation in eine Krise gerät und von einer jüngeren Generation eine neue Europapolitik durchgesetzt wird;

- schließlich die bekannteste Vorstellung von einem kontinuierlichen Voranschreiten der europäischen Integration vor allem durch produktive Krisen, in denen die Politik immer wieder gezwungen wird, neue Europakonzepte zu entwickeln.

Die Vorstellung von einer Spirale nach unten, einer fortwährenden Verschärfung der Krisen der europäischen Integration erhielt in den letzten Jahren einigen Zulauf. Aber die historische Entwicklung spricht nicht für sie. Die Krise der unmittelbaren Nachkriegszeit und die Krise der frühen 1970er Jahre hatten zwar jeweils einen anderen Charakter als die jüngste Krise, waren aber auf ihre Weise ähnlich schwere Krisen. Auch für die Vorstellung von Krisenzyklen, in denen jüngere Generationen von Europapolitkern ältere Generationen ablösen, gibt es keine starken Anhaltspunkte. Am ehesten entspricht der historischen Entwicklung das Konzept der produktiven Krise, allerdings unter den Einschränkungen, dass nicht jede Krise der europäischen Integration produktiv wurde und zudem manche Krisenlösungen die Ursache für die nächste Krise waren. Vor allem konnten Krisen nur aufgrund von bestimmten Voraussetzungen produktiv werden, die allerdings bisher in der Geschichte der 
europäischen Integration seit den späten 1940er und 1950er Jahren immer gegeben waren.

Diese Voraussetzungen sind:

- die grundsätzliche Unterstützung der europäischen Integration durch eine Mehrheit der Bevölkerung und der Eliten;

- die Überbrückung der internen Interessengegensätze durch Elitenetzwerke;

- die funktionierende deutsch-französische Zusammenarbeit in europäischen Entscheidungen;

- die allgemeine Akzeptanz der Leistungen der Europäischen Union und hohe Erwartungen in ihre zukünftige Leistungskraft.

\section{Parteiendemokratie in Europa}

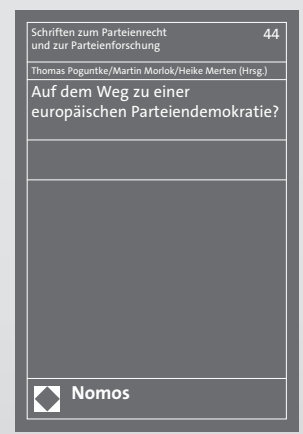

Den politischen Parteien kommt im gesamteuropäischen Willensbildungsprozess eine Schlüsselrolle zu, die allerdings bis heute von den nationalen Parteien besetzt wird. Um der Dynamik

\author{
Auf dem Weg zu \\ einer europäischen \\ Parteiendemokratie \\ Herausgegeben von Thomas \\ Poguntke, Martin Morlok und \\ Heike Merten \\ 2013, 226 S., brosch., 44,- $€$ \\ ISBN 978-3-8487-0595-5 \\ (Schriften zum Parteienrecht und \\ zur Parteienforschung, Bd. 44)
}

des europäischen Integrationsprozesses auch in Zukunft gerecht zu werden, muss der Weg zu einer europäischen Parteiendemokratie über die Europarteien geebnet werden. 